\title{
IRAS 05358+3543: Multiple outflows at the earliest stages of massive star formation
}

\author{
H. Beuther ${ }^{1}$, P. Schilke ${ }^{1}$, F. Gueth ${ }^{1,2}$, M. McCaughrean ${ }^{3}$, M. Andersen ${ }^{3}$, \\ T. K. Sridharan ${ }^{4}$, and K. M. Menten ${ }^{1}$ \\ 1 Max-Planck-Institut für Radioastronomie, Auf dem Hügel 69, 53121 Bonn, Germany \\ 2 Institut de Radio Astronomie Millimétrique, 300 rue de la Piscine, 38406 Saint Martin d'Hères, France \\ 3 Astrophysikalisches Institut Potsdam, An der Sternwarte 16, 14482 Potsdam, Germany \\ ${ }^{4}$ Harvard-Smithsonian Center for Astrophysics, 60 Garden Street, MS 78, Cambridge, MA 02138, USA
}

Received 2 November 2001 /Accepted 28 February 2002

\begin{abstract}
We present a high-angular-resolution molecular line and millimeter continuum study of the massive star formation site IRAS 05358+3543. Observations with the Plateau de Bure Interferometer in CO 1-0, $\mathrm{SiO} 2-1$ and $\mathrm{H}^{13} \mathrm{CO}^{+} 1-0$ reveal at least three outflows which cannot be separated in single-dish data. Observations at millimeter and sub-millimeter wavelengths from the IRAM $30 \mathrm{~m}$ telescope and the CSO provide additional information on the region. The most remarkable feature is a highly collimated (collimation factor $\sim 10$ ) and massive $\left(>10 M_{\odot}\right)$ bipolar outflow of $\sim 1 \mathrm{pc}$ length, which is part of a quadrupolar outflow system. The three observed molecular outflows forming the IRAS $05358+3543$ outflow system resemble, in structure and collimation, those typical of low-mass star-forming regions. They might therefore, just like low-mass outflows, be explained by shock entrainment models of jets. We estimate a mass accretion rate of $\sim 10^{-4} M_{\odot} / \mathrm{yr}$, sufficient to overcome the radiative pressure of the central object and to build up a massive star, lending further support to the hypothesis that massive star formation occurs similarly to low-mass star formation, only with higher accretion rates and energetics. In the millimeter continuum, we find three sources near the center of the quadrupolar outflow, each with a mass of 75-100 $M_{\odot}$. These cores are associated with a complex region of infrared reflection nebulosities and their embedded illuminating sources. The molecular line data show that $\mathrm{SiO}$ is found mostly in the outflows, whereas $\mathrm{H}^{13} \mathrm{CO}^{+}$traces core-like structures, though likely with varying relative abundances. Thermal $\mathrm{CH}_{3} \mathrm{OH}$ comprises both features and can be disentangled into a core-tracing component at the line center, and wing emission following the outflows. A CO line-ratio study (using data of the $J=1-0,2-1$ and $6-5$ transitions) reveals local temperature gradients.
\end{abstract}

Key words. molecular data - stars: early-type - stars: formation - stars: individual: IRAS $05358+3543-$ ISM: jets and outflows

\section{Introduction}

It is still not known whether the physical processes leading to massive stars are similar to those of low-mass star formation, or whether different processes are taking place. Classical star formation scenarios predict moderate accretion rates around $10^{-6}-10^{-5} M_{\odot} \mathrm{yr}^{-1}$ (Shu 1977), which are incapable of overcoming the radiation pressure for sources more massive than approximately $10 M_{\odot}$ (Wolfire \& Cassinelli 1987). Observations over the last decade showed that massive star formation occurs most likely in a clustered mode, and theories were proposed that advocate the coalescence of protostars in dense cluster centers to build up the most massive sources (Bonnell et al. 1998;

Send offprint requests to: H. Beuther, e-mail: beuther@mpifr-bonn.mpg.de
Stahler et al. 2000). In contrast, according to other theories following the classical accretion scenario, it is possible to form massive stars via enhanced accretion and disks (Jijina \& Adams 1996; Norberg \& Maeder 2000; Tan \& McKee 2002; Yorke 2002). For more details on massive star formation see recent reviews, e.g., Stahler et al. (2000), Richer et al. (2000), Churchwell (2000), and Kurtz et al. (2000).

Because molecular outflows give on large angular scales a wealth of information about the innermost part of star-forming regions, in recent years massive outflows have been investigated in more detail. Such outflow studies seemed to indicate a lower degree of collimation for massive outflows than known for low-mass sources. Collimation factors between 1 and 2 were reported as compared to low-mass outflows, where collimation factors 
up to 10 are found (e.g., Richer et al. 2000). Based on those observations new outflow scenarios were suggested, e.g., Churchwell (2000) proposed that massive outflows might be produced by deflection of infalling material from the central protostar. But the analysis of the data has not taken into account properly the spatial resolution of single-dish observations. Massive star formation sites are on the average far more distant (a few $\mathrm{kpc}$ ) than well known low-mass sources (a few hundred pc), and thus their angular sizes are smaller in spite of larger linear sizes. Recent statistical work on massive outflows with $11^{\prime \prime}$ resolution by Beuther et al. (2002b) shows that the average collimation - even with this spatial resolution is higher than previously thought. Additionally, Beuther et al. (2002b) find that low-mass correlations of outflow and core parameters continue up to the high-mass regime, suggesting that similar star formation processes are responsible for forming stars of all masses. But their data also indicate that even higher angular resolution is needed to disentangle real source and outflow structures, and thus detailed studies with $\mathrm{mm}$ interferometers are needed.

There have been recent interferometric studies of massive outflow sources, of which prominent examples are IRAS 20126+4104 (Cesaroni et al. 1997; Cesaroni et al. 1999) or G192.16-3.82 (Shepherd et al. 1998; Shepherd \& Kurtz 1999). While the outflow of IRAS 20126+4104 is rather collimated, G192.16-3.82 does not show high degrees of collimation, and Shepherd \& Kurtz (1999) propose as outflow mechanism a combination of a strong, wideangle wind with a weak jet.

In this paper, we present a detailed interferometric and single-dish study at mm wavelengths of an extremely young and deeply embedded high-mass protostellar object IRAS $05358+3543$, which is also known in the literature as S233IR. This source is part of a larger sample presented and discussed by Sridharan et al. (2002) and Beuther et al. (2002a,b,c).

IRAS $05358+3543$ has different peak positions in the IRAS $12 \mu \mathrm{m}$ and $100 \mu \mathrm{m}$ bands. While the position in the Point Source Catalog is based on the $12 \mu \mathrm{m}$ emission due to an infrared cluster, the $100 \mu \mathrm{m}$ emission peaks approximately $40^{\prime \prime}$ away to the north-east and traces the high-mass protostellar cluster we are focusing on (Fig. 1). As the mm dust continuum emission coincides with the $100 \mu \mathrm{m}$ peak, we will refer to that source as IRAS $05358+3543 \mathrm{~mm}$. Based on IRAS infrared data, we estimate the bolometric luminosity of the source to be $\sim 6300 L_{\odot}$, and the dust temperature to be around $47 \mathrm{~K}$ (Table 2, Sridharan et al. 2002). The first CO 10 observations of this region are reported by Snell et al. (1990), who detected a $15 M_{\odot}$ bipolar outflow and classify the source as a massive star formation site. The estimated distance of $1.8 \mathrm{kpc}$ is based on observations of the nearby HiI region S233 (Snell et al. 1990). As additional massive star formation and outflow signposts, Class II $\mathrm{CH}_{3} \mathrm{OH}$ (Menten 1991; Sridharan et al. 2002) and $\mathrm{H}_{2} \mathrm{O}$ maser emission (Tofani et al. 1995; Beuther et al. 2002c) is observed towards IRAS $05358+3543 \mathrm{~mm}$. Additionally,

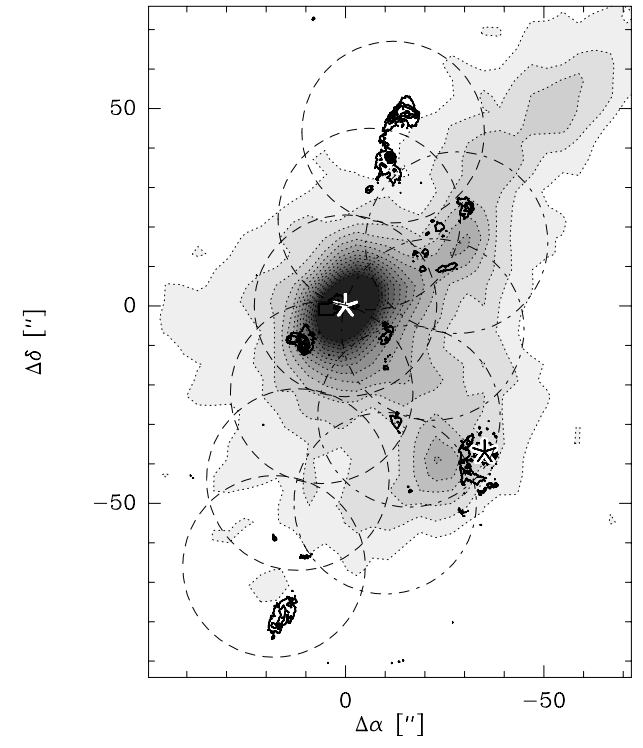

Fig. 1. The $1.2 \mathrm{~mm}$ bolometer image is plotted in grey-scale and dotted contours (the contour levels are $100 \mathrm{mJy}^{-1}$ beam $^{-1}$ to $1000 \mathrm{mJy}_{\text {beam }}{ }^{-1}$ in steps of $100 \mathrm{mJy}_{\text {beam }}{ }^{-1}$, in short 100(100)1000 mJy beam ${ }^{-1}$ ), and the $\mathrm{H}_{2}$ image (McCaughrean et al., in prep.) overlayed in contours (arbitrary but linear units). The dashed circles present the observed PdBI mosaic (the four dashed-dotted western fields were observed in only two configurations). The circular $\mathrm{H}_{2}$ feature at the right edge of the mosaic is due to the $12 \mu \mathrm{m} I R A S$ source (black star). The white star shows the mm continuum peak and reference center of the mosaic (RA[J2000] 05:39:13.0 and Dec[J2000] 35:45:54). The offsets are given in arcsecs from the center of the mosaic.

$\mathrm{OH}$ maser emission is found in the region with low spatial resolution (Wouterloot et al. 1988). As reported by Tofani et al. (1995) and confirmed by Sridharan et al. (2002), IRAS $05358+3543 \mathrm{~mm}$ does not show any $3.6 \mathrm{~cm}$ emission down to $1 \mathrm{mJy}$. Thus, based on the total luminosity of the region and a regular initial mass function, it is likely that we are witnessing a very early stage of massive star formation (see Sridharan et al. 2002). The spectral type of the most massive object of the cluster is likely between $\mathrm{B} 2$ and $\mathrm{B} 1\left(10 M_{\odot} \leq M \leq 13 M_{\odot}\right)$.

Porras et al. (2000), Yao et al. (2000) and Jiang et al. (2001) have published a series of near-infrared imaging and polarimetric studies of this region, showing that there are two young embedded stellar clusters, one associated with the $12 \mu \mathrm{m}$ source (age $\sim 3 \mathrm{Myr}$ ) and one with IRAS $05358+3543 \mathrm{~mm}$ (age $\leq 2 \mathrm{Myr}$ ). Narrow-band observations in the $v=1-0 \mathrm{~S}(1)$ line of molecular hydrogen at $2.122 \mu \mathrm{m}$ delineate at least two bipolar jets emanating from the younger cluster, and polarimetric imaging identifies the likely source of the outflows as being deeply embedded and undetected at near-infrared wavelengths (Yao et al. 2000; Jiang et al. 2001). In an accompanying paper, McCaughrean et al. (in prep.) present new, more sensitive, and higher-spatial-resolution near- and mid-infrared observations of the region, which reveal new information concerning the shocked $\mathrm{H}_{2}$ outflows and their driving sources. 
Table 1. Observation parameters of spectral line observations; observatories are the $30 \mathrm{~m}$ at Pico Veleta (PV), the Plateau de Bure Interferometer (PdBI), the Caltech Submillimeter Observatory (CSO), and the Very Large Array (VLA); quoted system temperatures $T_{\text {sys }}$ are average values, $\Delta v$ is the velocity resolution.

\begin{tabular}{|c|c|c|c|c|c|}
\hline & $\begin{array}{r}\text { freq. } \\
{[\mathrm{GHz}]}\end{array}$ & Obs & $\begin{array}{r}H P B W \\
{\left[{ }^{\prime \prime}\right]}\end{array}$ & $\begin{array}{r}T_{\text {sys }} \\
{[\mathrm{K}]} \\
\end{array}$ & $\begin{array}{r}v \\
{\left[\mathrm{~km} \mathrm{~s}^{-1}\right]}\end{array}$ \\
\hline CO $1-0$ & 115.27 & $\mathrm{PV}$ & 22 & 120 & 0.2 \\
\hline CO $1-0$ & 115.27 & $\mathrm{PdBI}$ & $4.1 \times 3.3$ & 300 & 0.4 \\
\hline $\mathrm{SiO} 2-1$ & 86.85 & PV & 29 & 85 & 3.5 \\
\hline $\mathrm{SiO} 2-1$ & 86.85 & $\mathrm{PdBI}$ & $5.8 \times 5.6$ & 150 & 0.3 \\
\hline $\mathrm{H}^{13} \mathrm{CO}^{+}$ & 86.75 & PV & 29 & 85 & 3.5 \\
\hline $\mathrm{H}^{13} \mathrm{CO}^{+}$ & 86.75 & PdBI & $5.8 \times 5.6$ & 150 & 0.3 \\
\hline${ }^{13} \mathrm{CO} 1-0$ & 110.20 & PV & 22 & 120 & 0.8 \\
\hline CO 2-1 & 230.54 & PV & 11 & 250 & 0.1 \\
\hline $\mathrm{CO} 6-5$ & 691.47 & $\mathrm{CSO}$ & 11 & 3000 & 0.02 \\
\hline $\mathrm{CH}_{3} \mathrm{OH}$ & 241.79 & PV & 11 & 250 & 0.1 \\
\hline
\end{tabular}

\section{Observations}

We have observed IRAS $05358+3543 \mathrm{~mm}$ in several tracers with different telescopes. Table 1 summarizes the observations described below.

\subsection{Plateau de Bure Interferometer (PdBI)}

IRAS $05358+3543 \mathrm{~mm}$ was observed with the IRAM Plateau de Bure millimeter array (Guilloteau et al. 1992) between August and October 1999 in two frequency setups $^{1}$. Five $15 \mathrm{~m}$ antennas equipped with dual-frequency receivers were used in three different configurations (4D1, $4 \mathrm{C} 2 \& 4 \mathrm{C} 2+\mathrm{N} 09$ ) with baselines ranging from $24 \mathrm{~m}$ to $180 \mathrm{~m}$. Only four antennas were used during the August observations. Because of the poor summer weather conditions, the quality of the $1 \mathrm{~mm}$ data was not satisfactory in both setups, and they were only used for phase corrections. The $3 \mathrm{~mm}$ receivers were tuned to $115.27 \mathrm{GHz}$ (USB) and 86.8 $\mathrm{GHz}$ (LSB) to cover the CO 1-0 line, and the SiO 2-1 and $\mathrm{H}^{13} \mathrm{CO}^{+} 1-0$ lines simultanously. The phase noise was lower than $30^{\circ}$ and atmospheric phase correction based on the $1.3 \mathrm{~mm}$ total power was applied. For continuum measurements, we placed two $160 \mathrm{MHz}$ correlator units in each band. Temporal fluctuations of amplitude and phase were calibrated with frequent observations of the quasars $2145+067$ and $0548+378$. The amplitude scale was derived from measurements of MWC349 and CRL618. We estimate the final flux density accuracy to be $\sim 15 \%$. The reference center is RA[J2000] 05:39:13.0 and Dec[J2000] $35: 45: 54$, and the $v_{\text {LSR }}$ is $-17.6 \mathrm{~km} \mathrm{~s}^{-1}$. Ten fields (see Fig. 1) were observed to cover the whole source, with the exception of the first configuration which covered only the 6 eastern fields.

1 The IRAM Plateau de Bure Interferometer is supported by INSU/CNRS (France), MPG (Germany) and IGN (Spain).
Table 2. Physical parameters of the observed region from $1.2 \mathrm{~mm}$ continuum data ( $M$ and $N_{\mathrm{H}_{2}}$, Sect. 3.1.1), IRAS ( $L$ and $T_{\text {dust }}$, Sridharan et al. 2002) and CO 2-1 observations ( $M_{\text {out }}, \dot{M}_{\text {out }}$ and dynamical age $t$, Sect. 3.1.2).

\begin{tabular}{lr}
\hline \hline$M\left[M_{\odot}\right]$ & 610 \\
$N_{\mathrm{H}_{2}}\left[\mathrm{~cm}^{-2}\right]$ & $4.0 \times 10^{24}$ \\
$L\left[L_{\odot}\right]$ & 6300 \\
$T_{\text {dust }}[\mathrm{K}]$ & 47 \\
$M_{\text {out }}\left[M_{\odot}\right]$ & 20 \\
$\dot{M}_{\text {out }}\left[M_{\odot} / \mathrm{yr}\right]$ & $6 \times 10^{-4}$ \\
$t[\mathrm{yr}]$ & 36000 \\
\hline
\end{tabular}

\subsection{Pico Veleta (PV)}

Line observations:

To obtain large scale information about IRAS $05358+3543 \mathrm{~mm}$, we used the IRAM $30 \mathrm{~m}$ telescope at Pico Veleta in the Sierra Nevada, Spain in April 1999 and mapped the whole region on-the-fly in the $\mathrm{CO} 2-1$, CO $1-0,{ }^{13} \mathrm{CO} 1-0, \mathrm{H}^{13} \mathrm{CO}^{+} 1-0$, SiO $2-1$ and $\mathrm{CH}_{3} \mathrm{OH} 5_{0}-4_{0}(A+)$ lines (near $241.79 \mathrm{GHz}$ ). Due to the continuous movement of the telescope during on-the-fly observations, spurious stripes in scanning direction (RA) are found in some maps (see Fig. 2). Except for CO 1-0 (observed later [May 2000] to add the short spacings, see Sect. 2.3) all the other lines were observed simultaneously, which guarantees the best alignment between the different maps. The on-the-fly coverages were done twice with $2 \mathrm{~s}$ integration time per dump and a $4^{\prime \prime}$ grid (i.e., Nyquist-sampling at $241 \mathrm{GHz}$ ). As backends we used the autocorrelator and two $1 \mathrm{MHz}$ filterbanks.

\section{Bolometer observations:}

The $1.2 \mathrm{~mm}$ single-dish continuum observations were conducted with the MAMBO array at the IRAM $30 \mathrm{~m}$ telescope. For details on the observations and analysis of this data see Beuther et al. (2002a).

\subsection{Merging the interferometric and single-dish data}

The IRAM 30 m data ( $\mathrm{CO} 1-0$, SiO $2-1$ and $\mathrm{H}^{13} \mathrm{CO}^{+} 1-0$ ) were used to derive short-spacing information and thereby complement the interferometric PdBI data. The algorithm used to derive the visibilities corresponding to each pointing center of the mosaic is described by Gueth et al. (1996). The single-dish and interferometer visibilities are subsequently processed together. Relative weighting has been chosen to minimize the negative side-lobes in the resulting dirty beam while keeping the highest angular resolution possible. Again images were produced using natural weighting, then a CLEAN-based deconvolution of the mosaic was performed. The final beam sizes are $4.7^{\prime \prime} \times 3.8^{\prime \prime}$ $\left(\mathrm{PA} 51^{\circ}\right)$ for $\mathrm{CO} 1-0$, and $5.9^{\prime \prime} \times 5.5^{\prime \prime}\left(\mathrm{PA} 65^{\circ}\right)$ for $\mathrm{H}^{13} \mathrm{CO}^{+}$ $1-0$ and $\mathrm{SiO} 2-1$. The beam size for the $115 \mathrm{GHz}$ continuum data, where only the PdBI data were used, is $4^{\prime \prime} \times 3^{\prime \prime}$ $\left(\mathrm{PA} 46^{\circ}\right)$. 


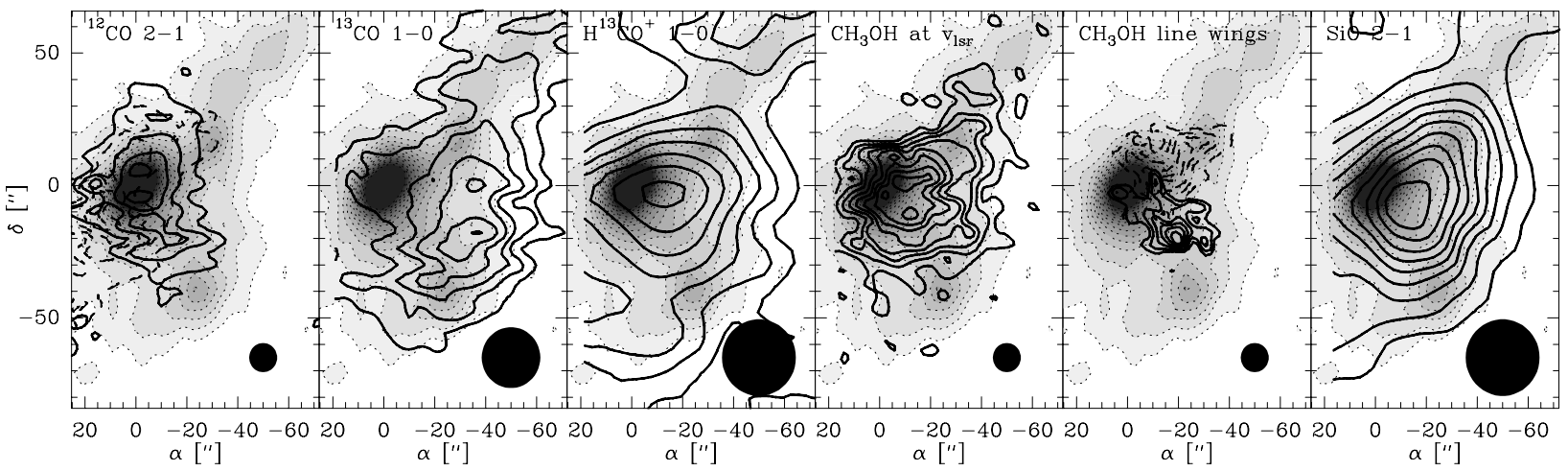

Fig. 2. IRAM $30 \mathrm{~m}$ single-dish observations. Each image shows the $1.2 \mathrm{~mm}$ continuum observations in grey-scale and dotted contours (levels 100(100)1000 mJy beam ${ }^{-1}$ ) with the molecular line observations overlayed in contours (labeled in the top left corners). We show the integrated intensity of ${ }^{13} \mathrm{CO} 1-0\left(v=[-22,-9] \mathrm{km} \mathrm{s}^{-1}\right.$, levels $\left.32(4) 52 \mathrm{~K} \mathrm{~km} \mathrm{~s}^{-1}\right), \mathrm{H}^{13} \mathrm{CO}^{+} 1-0$ $\left(v=[-26,-10] \mathrm{km} \mathrm{s}^{-1}\right.$, levels $\left.0.4(0.6) 5.2 \mathrm{~K} \mathrm{~km} \mathrm{~s}^{-1}\right)$ and $\mathrm{SiO} 2-1\left(v=[-26,-10] \mathrm{km} \mathrm{s}^{-1}\right.$, levels 0.6 (0.6)5.6 K km s $\left.\mathrm{K}^{-1}\right)$. The ${ }^{12} \mathrm{CO} 2-1$ map presents blue (solid contours, $v=[-32,-21] \mathrm{km} \mathrm{s}^{-1}$, levels $13(9) 40 \mathrm{~K} \mathrm{~km} \mathrm{~s}^{-1}$ ) and red (dashed contours, $v=$ $[-12,-4] \mathrm{km} \mathrm{s}^{-1}$, levels $13(9) 40 \mathrm{~K} \mathrm{~km} \mathrm{~s}^{-1}$ ) outflow emission. For thermal $\mathrm{CH}_{3} \mathrm{OH} 5_{0}-4_{0}(A+)$ emission at $241.79 \mathrm{GHz}$, we present one map for velocities near the line center $\left(v=[-17,-16] \mathrm{km} \mathrm{s}^{-1}\right.$, levels $\left.1.1(0.5) 4.6 \mathrm{~K} \mathrm{~km} \mathrm{~s}^{-1}\right)$ and one map for the line wings (blue: solid lines $v=[-20,-23] \mathrm{km} \mathrm{s}^{-1}$, levels $2.1(0.5) 4.6 \mathrm{~K} \mathrm{~km} \mathrm{~s}^{-1}$; red: dashed lines $v=[-14,-12] \mathrm{km} \mathrm{s}^{-1}$, levels $\left.1.1(0.5) 1.6 \mathrm{~K} \mathrm{~km} \mathrm{~s}^{-1}\right)$. The stretches in east-west direction, visible in the ${ }^{12} \mathrm{CO}$ and ${ }^{13} \mathrm{CO}$ maps, are artefacts of the on-the-fly observing mode. The beam size for each transition is shown in the bottom-right corners.

\subsection{Caltech Submillimeter Observatory (CSO)}

We used the $\mathrm{CSO}^{2} 10.4 \mathrm{~m}$ telescope to obtain a CO 6-5 map of IRAS $05358+3543 \mathrm{~mm}$ in the on-the-fly mode on December, 15th 1999. At this frequency, the angular resolution of the CSO is $11^{\prime \prime}$, well suited to be compared with the $\mathrm{CO} 2-1$ data of Pico Veleta. As backend we used the facility AOS. Further observation parameters are given in Table 1.

\section{Observational results}

\subsection{Single-dish observations}

\subsubsection{Dust continuum emission}

Figure 1 presents the $1.2 \mathrm{~mm}$ continuum image of IRAS $05358+3543 \mathrm{~mm}$. The main peak is at the IRAS $100 \mu \mathrm{m}$ peak position and has more extended emission to the north-west and the south-west. The latter subsource is directly next to the $12 \mu \mathrm{m}$ peak position, which is the center of a slightly older cluster (Porras et al. 2000) and can be interpreted as a remnant of this older starforming region. Additionally, there exists a north-western elongation in the dust map, which will be shown to be associated with molecular line emission (see Sect. 3.1.2).

Assuming that the mm continuum is mainly produced by optically thin dust emission with a grain emissivity index $\beta=2$, Beuther et al. (2002a) estimate the total gas mass $M$ to $\sim 600 M_{\odot}$ and the column density $N_{\mathrm{H}_{2}}$ to a few times $10^{24} \mathrm{~cm}^{-2}$ (Table 2), which shows that we are really dealing with a high-mass star formation site. The column density converts to a visual absorption magnitude

\footnotetext{
2 The CSO is operated by the California Institute of Technology under funding from the National Science Foundation, Grant No. AST 9980846.
}

$A_{\mathrm{v}}$ of $\sim 1000\left(A_{\mathrm{v}}=N_{\mathrm{H}_{2}} / 0.94 \times 10^{21}\right.$, Frerking 1982). If a central star has already ignited, its free-free emission could be quenched by the infalling core (Walmsley 1995).

\subsubsection{Molecular emission}

Figure 2 presents single-dish data obtained at the $30 \mathrm{~m}$ telescope on Pico Veleta. The ${ }^{12} \mathrm{CO} 2-1$ lines show selfabsorption at the line center (Fig. 3), and blue and red wing emission due to molecular outflows. Blue $(v=$ $\left.[-32,-21] \mathrm{km} \mathrm{s}^{-1}\right)$ and red $\left(v=[-12,-4] \mathrm{km} \mathrm{s}^{-1}\right){ }^{12} \mathrm{CO}$ wing emission maps were produced by integrating the wing-part of the spectra. It is difficult to disentangle bipolar structure in spite of the known outflow features presented by Porras et al. (2000), but the outflow lobes are centered at the mm core. As outlined in Sect. 3.2.2, the inclination angle of the outflow is low, and we use the mean intensities of the red and blue line-wing maps in the spatial area known from the Plateau de Bure observations for further outflow parameter determination. Opacity-corrected $\mathrm{H}_{2}$ column densities $N_{\mathrm{b}}$ and $N_{\mathrm{r}}$ in both outflow lobes can be calculated by assuming a constant ${ }^{13} \mathrm{CO} /{ }^{12} \mathrm{CO} 2-1$ line wing ratio throughout the outflow (Cabrit \& Bertout 1990). Choi et al. (1993) found an average ${ }^{13} \mathrm{CO} /{ }^{12} \mathrm{CO}$ $2-1$ line wing ratio around 0.1 in 7 massive star-forming regions, corresponding to a $\tau\left({ }^{13} \mathrm{CO} 2 \rightarrow 1\right)=0.1$. We adopt this value for our sample as well, and we assume $30 \mathrm{~K}$ as the average temperature in the outflow. The outflow mass $M_{\text {out }}$, the dynamical timescale $t$, and the mass entrainment rate $\dot{M}_{\text {out }}$ are calculated via:

$$
\begin{aligned}
& M_{\text {out }}=\left(N_{\mathrm{b}} \times \text { size }_{\mathrm{b}}+N_{\mathrm{r}} \times \text { size }_{\mathrm{r}}\right) m_{\mathrm{H}_{2}} \\
& t=\frac{r}{\left(v_{\text {maxb }_{\mathrm{b}}}+v_{\text {max }_{\mathrm{r}}}\right) / 2} \\
& \dot{M}_{\text {out }}=\frac{M_{\text {out }}}{t}
\end{aligned}
$$




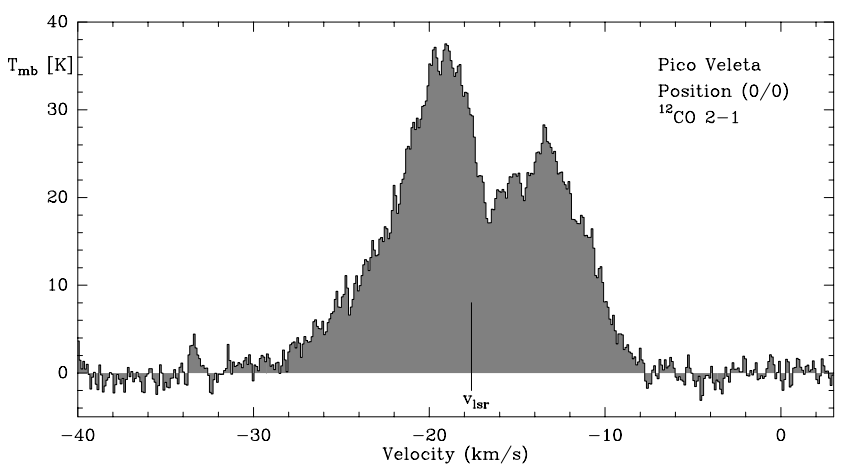

Fig. 3. The ${ }^{12} \mathrm{CO} 2-1$ spectrum observed with the $30 \mathrm{~m}$ at the center position of the main $\mathrm{mm}$ core. The system velocity $v_{\mathrm{LSR}}$ is $-17.6 \mathrm{~km} \mathrm{~s}^{-1}$.

where size $\mathrm{b}_{\mathrm{b}}$ and size $\mathrm{r}_{\mathrm{r}}$ are the areas of the blue and red outflow lobes, respectively, $m_{\mathrm{H}_{2}}$ the mass of the $\mathrm{H}_{2}$ molecule, and $v_{\text {max }_{\mathrm{b}}}$ and $v_{\text {max }_{\mathrm{r}}}$ the maximum velocities observed in each line wing. A more detailed description of how the outflow parameters are determined is given in Beuther et al. (2002b). According to Cabrit \& Bertout (1990) derived masses are accurate to a factor 2 to 4 , whereas the error in the determination of the dynamical parameters is higher, up to a factor 10 . The derived total outflow mass $M_{\text {out }}$ is around $20 M_{\odot}$ and the mass entrainment rate of molecular gas $\dot{M}_{\text {out }}$ approximately $6 \times 10^{-4} M_{\odot} \mathrm{yr}^{-1}$ (see Table 2). The latter value is derived dividing $M_{\text {out }}$ by the dynamical timescale $t$. As outlined in Beuther et al. (2002b), such a mass entrainment rate results in accretion rate estimates $\dot{M}_{\text {accr }}$ around $10^{-4} M_{\odot} \mathrm{yr}^{-1}$. These are lower limits, because the outflow is not strongly inclined to the plane of the sky (see Sect. 3.2.2), which minimizes the wing emission and by that the detectable (and separable) outflow emission. In spite of the low accuracy, these values are high and confirm the outflow to be massive.

All other molecular line maps presented in Fig. 2 peak at positions that are clearly offset from the main $\mathrm{mm}$ core. This is in contrast with the ${ }^{12} \mathrm{CO}$ emission, where not only the wing emission is centered on the main mm peak, but also the integrated emission (see the ${ }^{12} \mathrm{CO} 6-5$ map in Fig. 4). All data at Pico Veleta were taken simultaneously, thus pointing errors cannot produce these offsets. The ${ }^{13} \mathrm{CO} 1-0, \mathrm{H}^{13} \mathrm{CO}^{+}, \mathrm{SiO}$ and $\mathrm{CH}_{3} \mathrm{OH}$ images at the line center show a similar north-western elongation as the mm continuum map. Low spatial as well as spectral resolution of the $\mathrm{H}^{13} \mathrm{CO}^{+}$and $\mathrm{SiO}$ data prevents further analysis of these data, but the line wings of the $\mathrm{CH}_{3} \mathrm{OH}$ data show a bipolar distribution slightly shifted to the west with respect to the $\mathrm{CO}$ outflow, indicating that the $\mathrm{CH}_{3} \mathrm{OH}$ emission might trace a different outflow in the west (see Sect. 3.2.2).

\subsubsection{Temperature gradients}

Multi-line studies of rotationally excited $\mathrm{CO}$ provide a good tool to study temperature variations in molecular

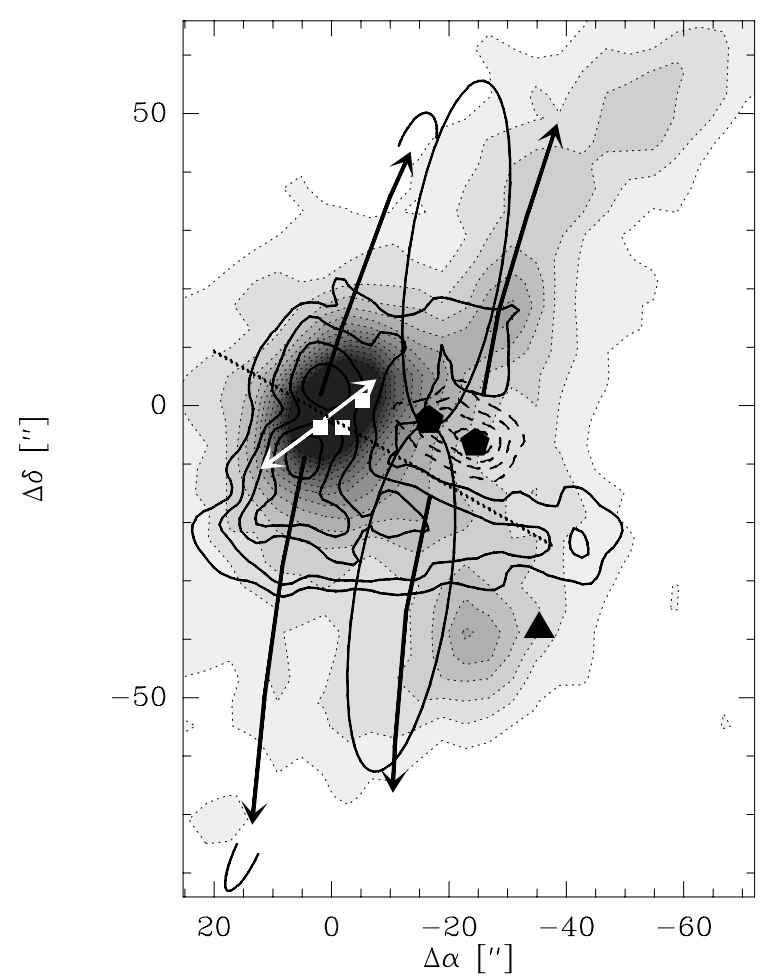

Fig. 4. The integrated CO $6-5$ emission in contours $(v=$

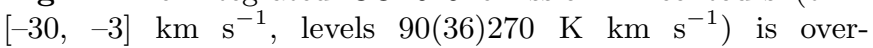
layed on the grey-scale $1.2 \mathrm{~mm}$ bolometer map (levels 100(100)1000 mJy beam ${ }^{-1}$ ). The dashed lines show the western $\mathrm{H}^{13} \mathrm{CO}^{+}$peak as outlined in Sect. 3.2.3 ( 1 channel at $-13.5 \mathrm{~km} \mathrm{~s}^{-1}$ is chosen, levels $25(25) 225 \mathrm{mJy}$ beam $\left.^{-1}\right)$. The overlayed raster of arrows and markers outlines the different outflows and sub-sources as described in Fig. 8.

clouds. In thermalized gas, where the local thermodynamical equilibrium (LTE) approximation applies, the low- $J$ CO 1-0 and 2-1 lines trace cooler material while the mid- $J$ CO $6-5$ line is sensitive to warmer molecular gas (e.g., Beuther et al. 2000; Hirano \& Taniguchi 2001). To get an idea of the temperature distribution in IRAS $05358+3543 \mathrm{~mm}$, we derived ${ }^{12} \mathrm{CO} 6-5 / 2-1$ and ${ }^{12} \mathrm{CO} 1-0 / 2-1$ ratio maps for the blue and red wing emission as defined in Sect. 3.1.2 (Fig. 5). All data are smoothed to the resolution of the CO 1-0 transition $\left(22^{\prime \prime}\right)$, even the $6-5 / 2-1$ ratio map to increase its signal-tonoise ratio.

In most regions rather uniform ratios around 0.5 are observed, but each of the ratio maps has a prominent region with line ratios larger than 1 . The blue $6-5 / 2-1$ ratios rise slightly east of the IRAS Position (named P1 and marked by an asterisk in Fig. 5 ), and the red $6-5 / 2-1$ ratios rise south-east of the main $\mathrm{mm}$ peak ( $\mathrm{P} 2$, triangle in Fig. 5), which is not prominent in the blue wings. Contrasting to the increases in the $6-5 / 2-1$ ratios around $\mathrm{P} 1$ and $\mathrm{P} 2$, the $1-0 / 2-1$ ratios are rather uniform around 0.5 throughout those regions, but they rise - independent of the line wings - around $30^{\prime \prime}$ west of the mm peak, where the mm emission drops significantly (P3, circle in Fig. 5). The blue $6-5 / 2-1$ ratios are lowest there. 


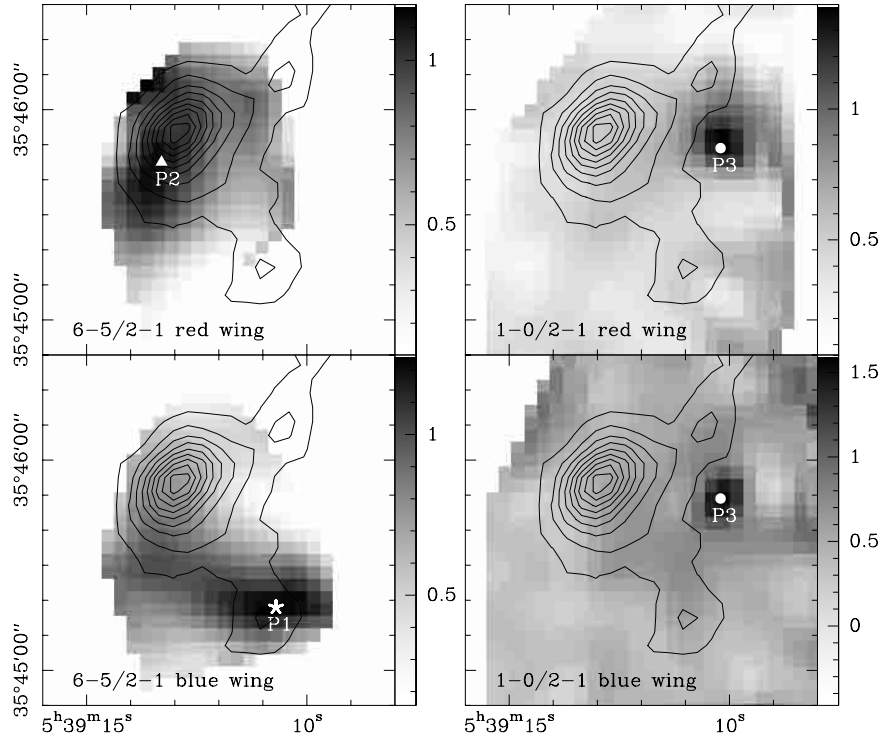

Fig. 5. Presented are in grey-scale the ratio maps of ${ }^{12} \mathrm{CO} 6-5 / 2-1$ (left) and ${ }^{12} \mathrm{CO} \quad 1-0 / 2-1$ (right) separated in blue (lower) and red (upper) wing emission (as defined in Sect. 3.1.2). In the blank white regions the signal to noise ratio is not sufficient to derive proper ratios. The contours show the $1.2 \mathrm{~mm}$ dust continuum map (levels 150(150)1050 mJy beam ${ }^{-1}$ ). The asterisk marks the hot-blue position ( $\mathrm{P} 1)$, the triangle the hot-red position (P2), and the circles the coldest position (P3).

As the average ${ }^{13} \mathrm{CO} /{ }^{12} \mathrm{CO} 2-1=0.1$ line wing ratio (Sect. 3.1.2) corresponds to a ${ }^{13} \mathrm{CO} 2-1$ opacity of $\approx 0.1$, the average ${ }^{12} \mathrm{CO} 2-1$ opacity in the wings is about 6 (Langer \& Penzias 1990). To quantify the temperatures in those regions, we ran several Large Velocity Gradient models (LVG) for optically thick $\mathrm{CO}$ emission $\left(\tau\left({ }^{12} \mathrm{CO} 2-1\right) \approx 6\right)$. The regions around $\mathrm{P} 1$ and $\mathrm{P} 2$ have average densities of a few times $10^{5} \mathrm{~cm}^{-3}$ (Beuther et al. 2002a), which is sufficient to thermalize even the $J=$ 6-5 transition. To thermalize the CO 2-1 transition, densities of $10^{4} \mathrm{~cm}^{-3}$ are sufficient, which is a reasonable assumption at position P3. Figure 6 presents the resulting line ratios versus the kinetic temperatures for the above estimated densities, as calculated via the LVG approximation. Obviously, increasing ${ }^{12} \mathrm{CO} 1-0 / 2-1$ ratios do indicate decreasing temperatures, whereas increasing ${ }^{12} \mathrm{CO} 6-5 / 2-1$ ratios are signposts of enhanced temperatures in such regions. The LVG calculations result in temperatures $\geq 80 \mathrm{~K}$ at $\mathrm{P} 1$ and $\mathrm{P} 2$, and temperatures below $20 \mathrm{~K}$ around P3.

\subsection{Interferometric observations}

\subsubsection{The main core at high resolution}

Zooming in the main single-dish $\mathrm{mm}$ core with the PdBI at $2.6 \mathrm{~mm}$ at a spatial resolution of $4^{\prime \prime} \times 3^{\prime \prime}(7000 \times 5000 \mathrm{AU})$, it resolves into three massive sub-sources with separations between $4^{\prime \prime}$ and $6^{\prime \prime}$. We label those three sources $\mathrm{mm} 1, \mathrm{~mm} 2$ and $\mathrm{mm} 3$ (see Fig. 7). The major and minor
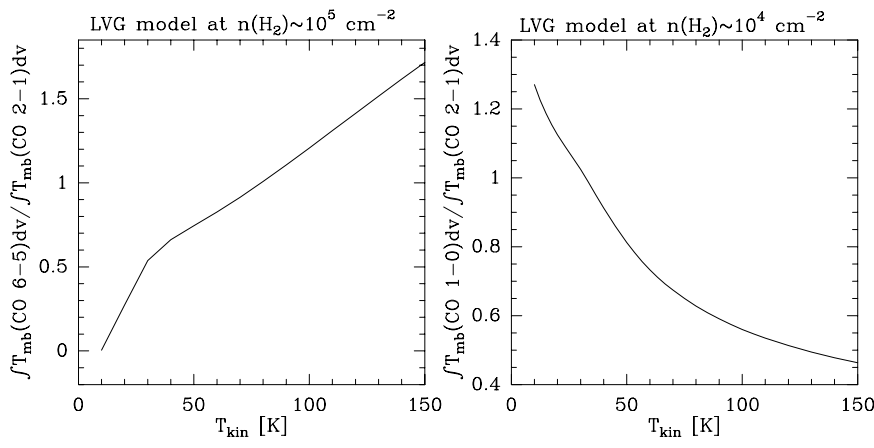

Fig. 6. CO ratios versus kinetic temperatures calculated via an LVG model for optical thick CO emission $\left(\tau\left({ }^{12} \mathrm{CO} 2-1\right) \approx 6\right)$. The left panel shows the ${ }^{12} \mathrm{CO} 6-5 / 2-1$ ratio at densities of $10^{5} \mathrm{~cm}^{-3}$, and the right panel presents the ${ }^{12} \mathrm{CO} 1-0 / 2-1$ ratio at densities of $10^{4} \mathrm{~cm}^{-3}$.

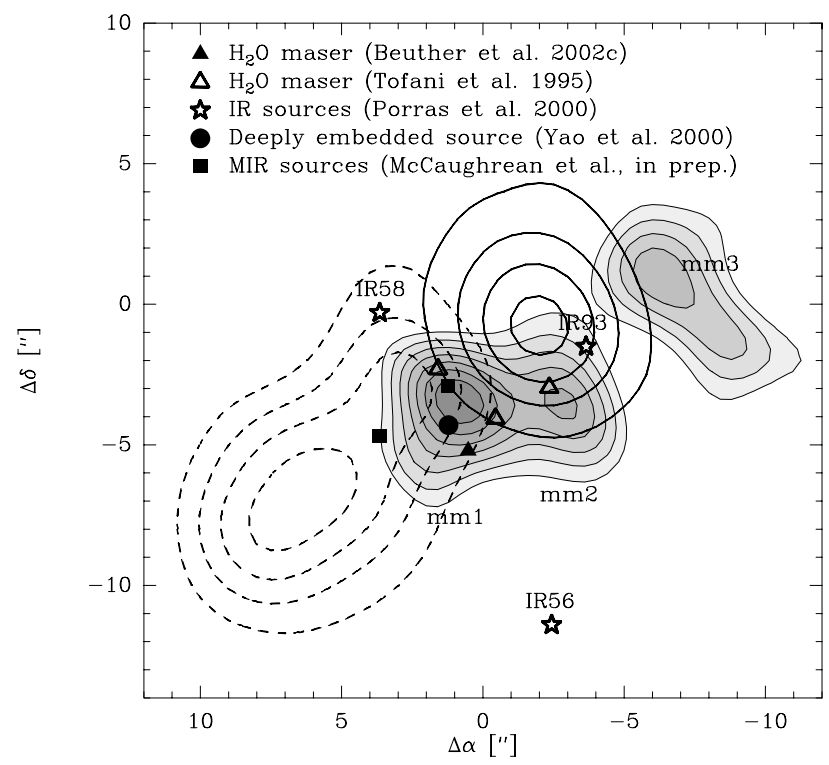

Fig. 7. The grey-scale shows the $3 \mathrm{~mm}$ continuum emission (mm1, mm2, mm3; levels 75(25)200 mJy beam ${ }^{-1}$ ) with the CO 1-0 high-velocity outflow $(\mathcal{B})$ overlayed in contours (blue: solid lines $v=[-43,-29] \mathrm{km} \mathrm{s}^{-1}$, levels $0.5(1) 3.5 \mathrm{Jy} \mathrm{beam}^{-1} \mathrm{~km} \mathrm{~s}^{-1}$; red: dashed lines $v=$ $[-9,0] \mathrm{km} \mathrm{s}^{-1}$, levels $\left.0.8(0.8) 4 \mathrm{Jy}^{\text {beam }}{ }^{-1} \mathrm{~km} \mathrm{~s}^{-1}\right)$.

core sizes were determined by fitting two-dimensional Gaussian to the data, the integrated emission is derived within approximately these areas. Assuming optically thin dust emission, masses and column densities presented in Table 3 were calculated at $30 \mathrm{~K}$ (for more details on the calculations see Beuther et al. 2002a). Source mm1 coincides within less than $1^{\prime \prime}$ with the main mid-infrared source (at $11.7 \mu \mathrm{m}$ ) presented by McCaughrean et al. (in prep.) as well as with the deeply embedded source found by Yao et al. (2000) and Jiang et al. (2001) in their $K$-band polarimetric imaging study.

Three $\mathrm{H}_{2} \mathrm{O}$ maser features found by Tofani et al. (1995) are associated with $\mathrm{mm} 1$, while one is associated with mm2. In 1999, Beuther et al. (2002c) detected only one of the previously known $\mathrm{H}_{2} \mathrm{O}$ maser feature south of $\mathrm{mm} 1$ (Fig. 7). 


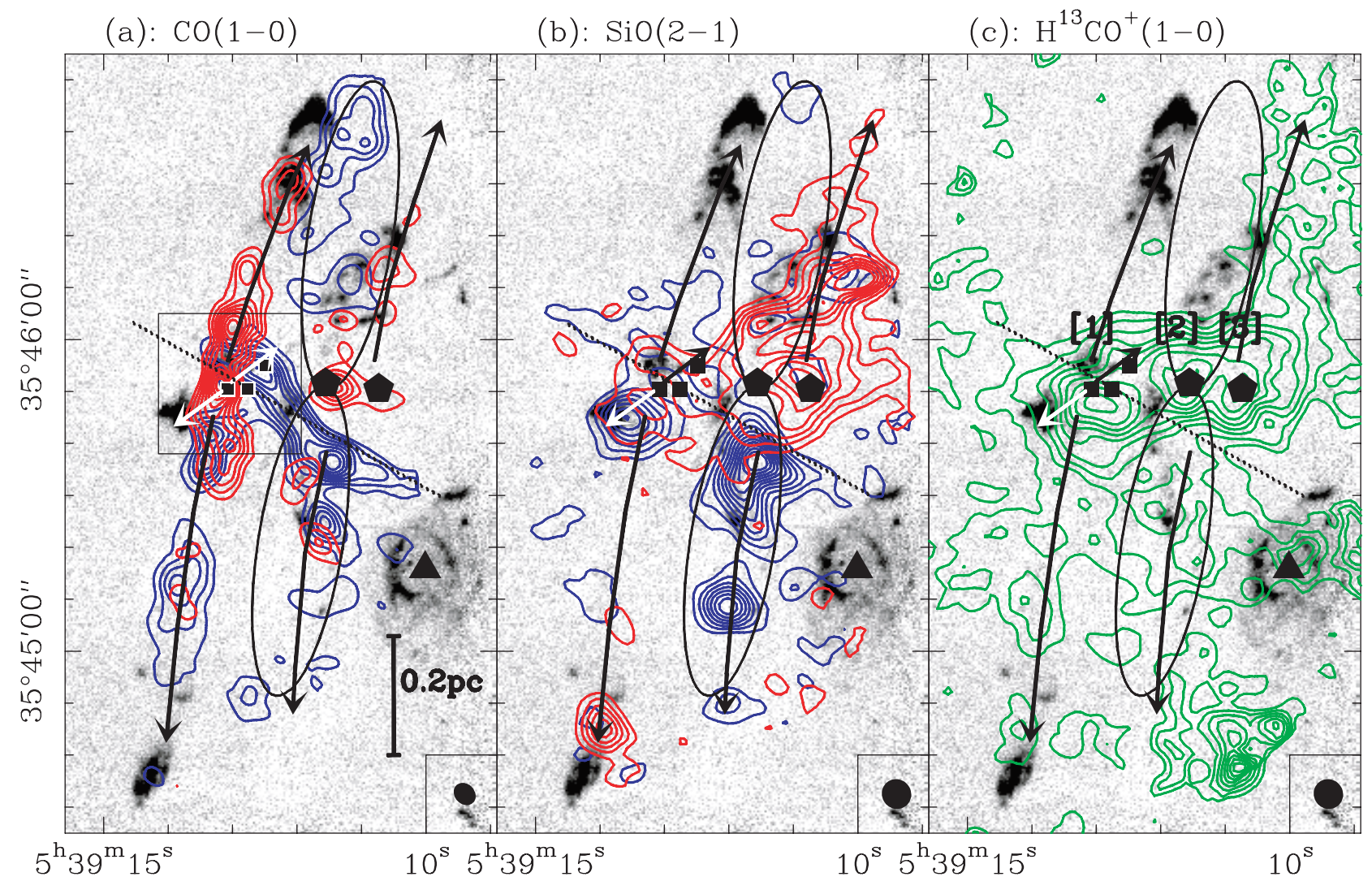

Fig. 8. Presented are the PdBI observations as contour overlays on the grey-scale $\mathrm{H}_{2}$ data (McCaughrean et al., in prep.). a) Outflow $(\mathcal{A})$ : CO $1-0$, red wing emission $\left(v=[-14,-7] \mathrm{km} \mathrm{s}^{-1}\right.$, levels $5(10) 95 \%$ from the peak intensity, PdB data only), and blue wing emission $\left(v=[-30,-21] \mathrm{km} \mathrm{s}^{-1}\right.$, levels $10(10) 90 \%$ from the peak intensity, PdB data only), the box marks the region shown in Fig. 7. b) Outflow $(\mathcal{C})$ : $\mathrm{SiO} 2-1$, red wing emission $\left(v=[-15.5,-8.5] \mathrm{km} \mathrm{s}^{-1}\right.$, levels $\left.0.15(0.15) 1.15 \mathrm{Jy} \mathrm{beam}^{-1} \mathrm{~km} \mathrm{~s}^{-1}\right)$, and blue wing emission $\left(v=[-28.5,-20.5] \mathrm{km} \mathrm{s}^{-1}\right.$, levels $0.15(0.15) 1.75 \mathrm{Jy} \mathrm{beam}^{-1} \mathrm{~km} \mathrm{~s}^{-1}$; PdBI data with short spacings from $\mathrm{PV}) . \mathbf{c}) \mathrm{H}^{13} \mathrm{CO}^{+} 1-0$, the integrated intensities are shown in green contours $\left(v=[-20.5,-10.5] \mathrm{km} \mathrm{s} \mathrm{s}^{-1}\right.$, levels $0.18(0.18) 1.08 \mathrm{Jy}_{\text {beam }}^{-1} \mathrm{~km} \mathrm{~s}^{-1}$, PdBI data with short spacings from PV). The numbers in brackets label the three $\mathrm{H}^{13} \mathrm{CO}^{+}$sources, the beams are shown each time at the bottom-right. In all images, the arrows outline the three main outflows, $(\mathcal{A})$ : long north-south arrows in the east, $(\mathcal{B})$ : short arrows in the east, and $\left(\mathcal{C}_{\text {bent }}\right)$ : long arrows in the west. The ellipses outline the second interpretation of the western outflow $\left(\mathcal{C}_{\text {cavity }}\right)$, and the dotted east-west line shows the speculative forth outflow. The three squares represent the mm sources, the diamonds locate the $\mathrm{H}^{13} \mathrm{CO}^{+}$peaks [2] and [3], and the triangle marks the $12 \mu \mathrm{m}$ IRAS position.

Table 3. Parameters derived for the PdBI mm cores: positions, major and minor core sizes, peak and integrated intensities, and masses and column densities.

\begin{tabular}{lrrr}
\hline \hline & $\mathrm{mm} 1$ & $\mathrm{~mm} 2$ & $\mathrm{~mm} 3$ \\
\hline RA [J2000.0] & $5: 39: 13.08$ & $5: 39: 12.78$ & $5: 39: 12.49$ \\
Dec [J2000.0] & $35: 45: 50.5$ & $35: 45: 50.6$ & $35: 45: 55.2$ \\
maj.×min. ["] & $5.6 \times 4.5$ & $5.6 \times 4.1$ & $6.1 \times 4.1$ \\
peak [mJy/beam] & 23 & 16 & 16 \\
int. [mJy] & 30 & 22 & 23 \\
$M\left[M_{\odot}\right]$ & 100 & 73 & 77 \\
$N_{\mathrm{H}_{2}}\left[10^{24} \mathrm{~cm}^{-2}\right]$ & 5.1 & 3.5 & 3.5 \\
\hline
\end{tabular}

The $\mathrm{CH}_{3} \mathrm{OH}$ maser feature is located near the $\mathrm{mm}$ cores as well, but the position is based on single-dish observations (Menten 1991) and not accurate enough for a closer interpretation.

Porras et al. (2000) found 3 infrared sources (IR56, IR58 and IR93 in Fig. 7) that are all offset from mm1 and mm2. Yao et al. (2000) and Jiang et al. (2001) then showed that the emission from IR58 and IR93 is highly polarized, and thus both are not independent sources but reflection nebulae powered by $\mathrm{mm} 1$.

\subsubsection{Three (at least) bipolar outflows}

The most striking features of IRAS $05358+3543 \mathrm{~mm}$ are the three outflows observed with the Plateau de Bure Interferometer in $\mathrm{CO}$ and $\mathrm{SiO}$ (Fig. 8).

\section{A large scale highly collimated $\mathrm{CO}$ outflow $(\mathcal{A})$}

Figure 8 a shows a highly collimated large scale $(\approx 1 \mathrm{pc})$ molecular outflow observed in $\mathrm{CO}$ in the east of the 
region. We present the PdBI data without merging the single-dish data in Fig. 8a, because the spatial filtering properties of the interferometer help to isolate the flow from the ambient gas (the combined data are presented in Sect. 3.2.4). To highlight the differences between outflow $(\mathcal{A})$ and the high-velocity outflow $(\mathcal{B})$, which is presented in the next paragraph, we used slightly different velocity windows compared to the single-dish image (Fig. 2). Outflow $(\mathcal{A})$ is slightly bent and oriented from north to south terminating in $\mathrm{H}_{2}$ bow shocks. This corresponds to one of the two outflows discussed by Porras et al. (2000) and by McCaughrean et al. (in prep.) based on $\mathrm{H}_{2}$ data. To quantify the degree of collimation, we divide the length of the outflow by its width, which results in a collimation factor of 10 . A number of emission peaks along the outflow show red and blue emission in CO and $\mathrm{SiO}$ (Figs. 8a,b), which is a typical feature for expanding bow shocks near the plane of the sky. Thus, we conclude that the outflow is not strongly inclined to the plane of the sky. Porras et al. (2000) claim that IR93 is powering the outflow, but Yao et al. (2000) and Jiang et al. (2001) show that IR93 is highly polarized and thus not a separate source. Our mm data, the mid-infrared images by McCaughrean et al. (in prep.), and the polarimetric images by Yao et al. (2000) and Jiang et al. (2001) strongly suggest that a protostar within $\mathrm{mm} 1$ is the powering source of outflow $(\mathcal{A})$.

\section{A high-velocity $\mathrm{CO}$ outflow $(\mathcal{B})$}

A second bipolar outflow is seen in $\mathrm{CO}$ at high velocities relative to the system velocity (Fig. 7 , blue lobe $v=$ $[-43,-29] \mathrm{km} \mathrm{s}^{-1}$, red lobe $\left.v=[-9,0] \mathrm{km} \mathrm{s}^{-1}\right)$. As Fig. 7 shows, this outflow is driven most likely by a source within the same protostellar condensation as outflow $(\mathcal{A})$, and both outflows together form a quadrupolar system, inclined by a position angle $\mathrm{PA}$ of $\approx 40^{\circ}$. McCaughrean et al. (in prep.) differently speculate that the second mid-infrared source $2^{\prime \prime}$ east might be the powering source. Blue and red lobes are clearly resolved by the PdBI with the red one to the south-east and the blue one to the north-west. The high-velocity outflow corresponds to the second outflow detected in $\mathrm{H}_{2}$ emission by Porras et al. (2000) and further discussed by McCaughrean et al. (in prep.). It has a collimation factor around 6 (estimated from combined $\mathrm{CO}$ and $\mathrm{H}_{2}$ data). At the tip of the south-eastern lobe, shocked $\mathrm{SiO}$ and $\mathrm{H}_{2}$ emission is observed (Figs. 8a,b). This is also the position of the warm region P2 (see Sect. 3.1.3), which suggests that the temperature increase may be caused by shock interaction of the outflow with the ambient medium.

\section{A third outflow mainly observed in $\mathrm{SiO}(\mathcal{C})$}

Further to the west, a third outflow is observed mainly in $\mathrm{SiO} 2-1$ on large scales in north-south direction (Fig. 8b). Red and blue lobes are resolved with three symmetric bullet-like features in each lobe. But as the region is very
Table 4. Outflow parameters from the merged PdBI and Pico Veleta observations.

\begin{tabular}{lrrrr}
\hline \hline & $\begin{array}{r}M_{\text {out }} \\
{\left[M_{\odot}\right]}\end{array}$ & $\left.\begin{array}{r}\dot{M}_{\text {out }} \\
{\left[10^{-4}\right.}\end{array} M_{\odot} \mathrm{yr}^{-1}\right]$ & $\begin{array}{r}t \\
{[\mathrm{yr}]}\end{array}$ \\
\hline$(\mathcal{A})+(\mathcal{B})$ & 9.6 & 2.6 & 37000 \\
$\left(\mathcal{C}_{\text {bent }}\right)$ & 4.4 & 1.4 & 31000 \\
All emission & 16.9 & 4.6 & 37000 \\
\hline
\end{tabular}

complicated, we present two alternate scenarios for the observed outflow features in this region.

(1) Outflow $\left(\mathcal{C}_{\text {bent }}\right)$ : the first hypothesis of outflow structure is outlined by the two western arrows in Fig. 8 . This way, the outflow shows the same bending as the large scale $\mathrm{CO}$ outflow $(\mathcal{A})$ in the east, and it follows to a large degree the mm emission as outlined in Fig. 4. CO emission is found there with the same bending structure (Fig. 8a). The collimation factor of this outflow is around 3 , and the powering source is possibly the western $\mathrm{H}^{13} \mathrm{CO}^{+}[3]$ core (Sect. 3.2.3).

(2) Outflow $\left(\mathcal{C}_{\text {cavity }}\right)$ : the other possible interpretation of the observation is sketched by the two ellipses presented in Fig. 8. This way, the northern $\mathrm{SiO}$ emission is not tracing the main part of the outflow, but rather the western cavity wall. The northern elongated $\mathrm{CO}$ feature (Fig. 8a), which is not accounted for in the previous scenario, could then represent the bow shock at the tip of the cavity. A problem with this interpretation is that the $\mathrm{SiO}$ emission in the north is mainly red-shifted with regard to the system velocity, whereas the northern $\mathrm{CO}$ emission is on the blue side of the spectrum with a difference of approximately $10 \mathrm{~km} \mathrm{~s}^{-1}$. A possible solution of this discrepancy is that the outflow is in the plane of the sky at the front side of the dense gas traced by the $1.2 \mathrm{~mm}$ dust continuum emission. Thus, the $\mathrm{SiO}$ emission is produced at the backside of the cavity, where the outflow interacts with the dense gas, and the material moves away from the observer. The blue CO feature then could be produced in the final expanding bow shock, when the outflow interacts with less dense gas at the tip of the flow, which is in front of the outflow and thus pushed towards the observer, producing blue-shifted emission. In this interpretation, not $\mathrm{H}^{13} \mathrm{CO}^{+}[3]$ but rather the $\mathrm{H}^{13} \mathrm{CO}^{+}$core [2] is the center of the outflow (Sect. 3.2.3), and the bending structure, especially of the northern lobe, is less prominent. The collimation factor of this structure is approximately 3 as well.

The southern part of the outflow seems to be less complicated and can be interpreted in both scenarios. At the edge of the more evolved IRAS $12 \mu \mathrm{m}$ cluster, we find the hot blue region $\mathrm{P} 1$ described in Sect. 3.1.3. The temperature increase of the outflowing gas in this region could be caused by UV heating of the adjacent more evolved cluster. We point out that the details of the morphological interpretation of outflow $(\mathcal{C})$ have no bearing on the overall energetics of the whole outflow system, which is dominated by outflow $(\mathcal{A})$. 


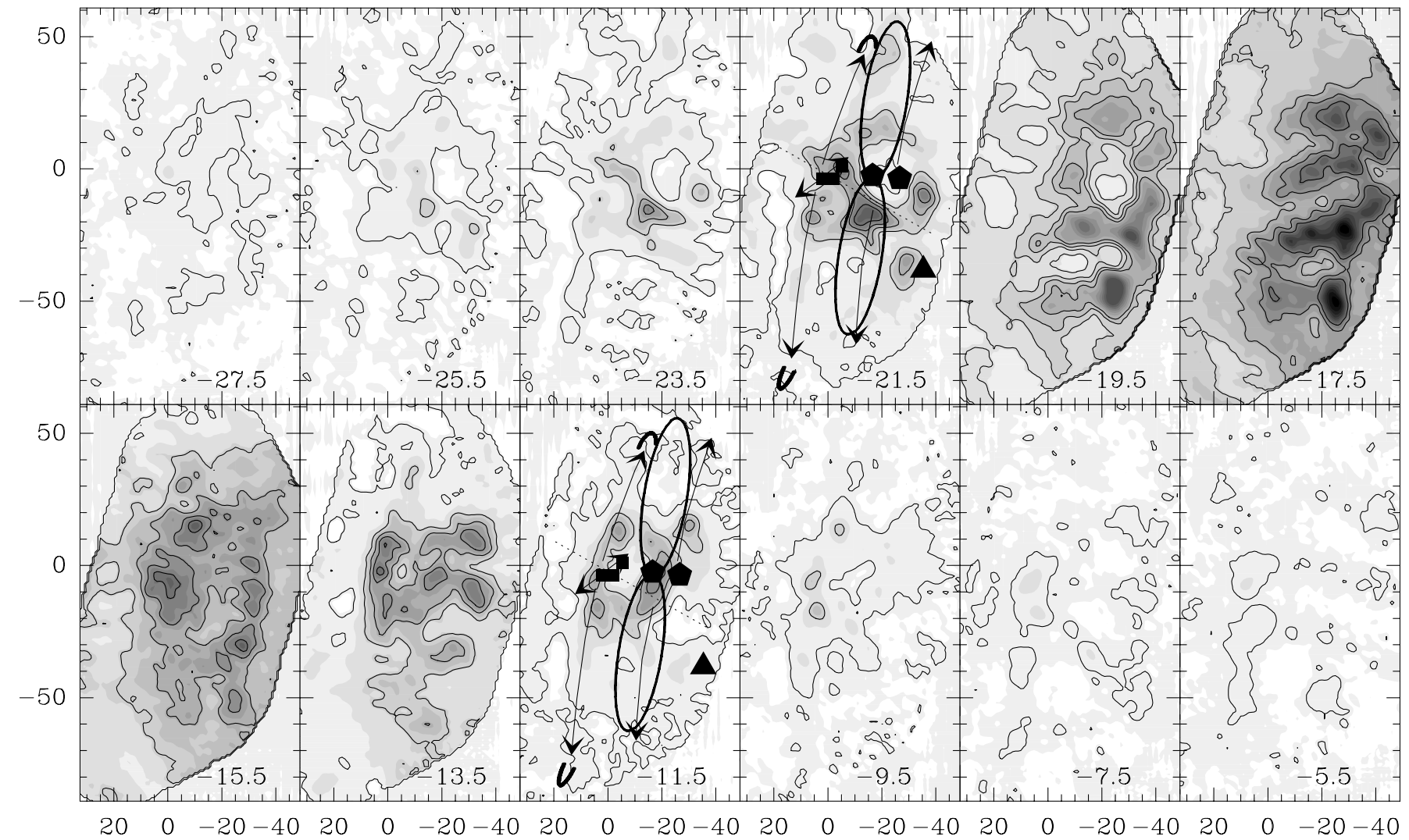

Fig. 9. Channel map of the region in CO 1-0, Plateau de Bure data merged with short spacings from Pico Veleta. The grey-scale levels are between 0 and $10 \mathrm{Jy} \mathrm{beam}^{-1} \mathrm{~km} \mathrm{~s}^{-1}$, and the contours range between 0.1 and 5.1 (by 1 ) $\mathrm{Jy} \mathrm{beam}^{-1} \mathrm{~km} \mathrm{~s}^{-1}$. The bottom-right corner shows the centroid velocity of each channel. The overlayed arrows and markers in two channels are the same as in Fig. 8.

\section{Outflow parameters}

We calculated the outflow parameters from the merged CO Plateau de Bure and $30 \mathrm{~m}$ data with the same assumptions and within the same velocity range as outlined in Sect. 3.1.2. The derived values (Table 4) agree reasonably well with the single-dish results (Table 2), and make us confident that the orders of magnitude are correct. For this calculations we cannot disentangle properly the contributions of the outflows $(\mathcal{A})$ and $(\mathcal{B})$, thus the derived values include both outflows. But from the spatial extent of both outflows, it becomes clear that most of the outflowing mass and of the mass entrainment rate is due to the large scale flow $(\mathcal{A})$. The value of outflow $(\mathcal{C})$ is calculated for the morphologically bent interpretation $\left(\mathcal{C}_{\text {bent }}\right)$.

\section{Additional features}

Are there even more outflows? We tentatively identify one more outflow oriented in east-west direction. Molecular line emission of $\mathrm{CO}$ and $\mathrm{SiO}$ shows extensions in that direction, in the interferometric data as well as with the single-dish observations (dotted east-west line in Figs. 4, 8 , and 9). Furthermore, at the western end of these molecular extensions, $\mathrm{H}_{2}$ emission is detected. One of the $\mathrm{mm}$ sources $\mathrm{mm} 2$ or $\mathrm{mm} 3$ might be the center of this tentative flow. Finally, we note that even more outflows are indicated in the $\mathrm{H}_{2}$ data of McCaughrean et al. (in prep.).

\subsection{3. $\mathrm{H}^{13} \mathrm{CO}^{+}$emission}

The PdBI data of $\mathrm{H}^{13} \mathrm{CO}^{+} 1-0$ show three peaks in eastwest orientation, and more extended emission to the north and south. One $\mathrm{H}^{13} \mathrm{CO}^{+}$peak $\left(\mathrm{H}^{13} \mathrm{CO}^{+}[1]\right)$ is associated with the three mm continuum peaks ( $\mathrm{mm} 1$ to $\mathrm{mm} 3$ ). The other $\mathrm{H}^{13} \mathrm{CO}^{+}$peaks [2] and [3] are both close to the center of outflow $(\mathcal{C})$, but we cannot determine with certainty, which of the two is the powering source of this flow (Sect. 3.2.2). Assuming local thermodynamic equilibrium, an $\mathrm{HCO}^{+}$abundance of $1 \times 10^{-9}$ (van Dishoeck 1993) and a $\mathrm{C}$ to ${ }^{13} \mathrm{C}$ ratio of 67 (Langer \& Penzias 1990), we can estimate the approximate masses of the $\mathrm{H}^{13} \mathrm{CO}^{+}$clumps, which are listed in Table 5. The temperatures decrease to the west according to the line ratio results found in Sect. 3.1.3.

The three $\mathrm{H}^{13} \mathrm{CO}^{+}$clumps are aligned in east-west direction with similar fluxes in each clump. Morphologically, this is different from the mm continuum emission which decreases towards the west (see Fig. 1). Therefore, the $\mathrm{H}^{13} \mathrm{CO}^{+}$peaks correspond only weakly to column density and mass concentrations traced by the $\mathrm{mm}$ continuum. 
Table 5. Masses of the $\mathrm{H}^{13} \mathrm{CO}^{+}$clumps at the given temperatures. The temperatures decrease to the west (Sect. 3.1.3).

\begin{tabular}{lccc}
\hline \hline $\mathrm{H}^{13} \mathrm{CO}^{+}$ & {$[1]$} & {$[2]$} & {$[3]$} \\
\hline $\operatorname{mass}\left[M_{\odot}\right]$ & 19 & 6 & 8 \\
$T[\mathrm{~K}]$ & 30 & 20 & 15 \\
\hline
\end{tabular}

As we do not have other high-resolution molecular line observations, it is difficult to discriminate between abundance and density variations, and it is probable that the $\mathrm{H}^{13} \mathrm{CO}^{+}$peaks [2] and [3] are caused by an interplay of different processes. The mass of $\mathrm{H}^{13} \mathrm{CO}^{+}[1]$ is about an order of magnitude below the value we derive from the $\mathrm{mm}$ continuum (Tables 2 and 3). Thus, in this region $\mathrm{H}^{13} \mathrm{CO}^{+}$is likely depleted. Remarkably, we detect no mm source near $\mathrm{H}^{13} \mathrm{CO}^{+}[2]$ and [3] and therefore at the center of outflow $(\mathcal{C})$. The $2.6 \mathrm{~mm} \mathrm{PdBI}$ data show at the $\mathrm{H}^{13} \mathrm{CO}^{+}[3]$ position just a peak at the $2 \sigma$ level, and our $3 \sigma \mathrm{mm}$ sensitivity corresponds to a mass limit of $\sim 50 M_{\odot}$ (at $\left.15 \mathrm{~K}\right)$. Even by a factor 5 enhanced $\mathrm{H}^{13} \mathrm{CO}^{+}$abundances result in lower mass estimates based on the $\mathrm{H}^{13} \mathrm{CO}^{+}$data, and the clump stays undetected in the mm continuum with our sensitivity limit. This is also the region P3, where the temperature decreases significantly according to the CO $1-0 / 2-1$ ratios (Sect. 3.1.3), and where only weak emission is observed in the integrated CO 6-5 map (Fig. 4).

\subsubsection{Channel maps of the $\mathrm{CO}$ data}

In Figure 8a we show for clarity the CO 1-0 data from the interferometer only, since the large-scale flow is more pronounced than in the merged data. Figure 9 now presents a channel map of the Plateau de Bure data merged with short spacings obtained at Pico Veleta. The eastern large scale outflow is still visible (e.g., channels at $-25.5,-23.5$, -7.5 and $\left.-5.5\left[\mathrm{~km} \mathrm{~s}^{-1}\right]\right)$, and new features appear by adding the short spacings due to bright extended emission at velocities close to the system velocity.

The ring-like structures seen in a number of channels are not artefacts caused by the merging with the interferometric data, because the ring is also seen in the ${ }^{12}$ CO $6-5$ single-dish data (Fig. 4). At $-21.5 \mathrm{~km}^{-1}$, the ring-like structure seems to surround the position of the $\mathrm{H}^{13} \mathrm{CO}^{+}[3]$ peak, which suggests that self absorption could cause the ring-like structure. But as the center of this structure moves spatially between the different channels, the ring might be produced by different overlapping outflows. The east-west structure at $-23.5 \mathrm{~km} \mathrm{~s}^{-1}$ has the same orientation as the putative fourth flow, which supports our idea of another outflow.

\subsection{Interpretation of single-dish maps with $P d B I$ data}

Some of the puzzling single-dish features outlined in Sect. 3.1.2 can be explained with the high-resolution data.

Being near the plane of the sky, outflow $(\mathcal{A})$ is difficult to resolve from the ambient gas in the single-dish data, and the outflows are far better visible with the Plateau de Bure Interferometer alone. Its higher spatial resolution is capable of resolving different flows spatially, whereas the interferometric feature of filtering out large scale uniform emission makes outflow $(\mathcal{A})$ a very prominent structure (Fig. 8a).

Interestingly, the offsets between the molecular line peaks of $\mathrm{H}^{13} \mathrm{CO}^{+}, \mathrm{SiO}$, and $\mathrm{CH}_{3} \mathrm{OH}$, and the $1.2 \mathrm{~mm}$ continuum peak are based on different physical processes in spite of their similar spatial distribution in the single-dish observation (Fig. 2). In the case of $\mathrm{H}^{13} \mathrm{CO}^{+}$, the PdBI data resolve the single-dish map into three sub-sources with an extension along the north western $1.2 \mathrm{~mm}$ continuum ridge, which makes $\mathrm{H}^{13} \mathrm{CO}^{+}$being at least partly a tracer of core-structure, though likely with varying relative abundances (Sect. 3.2.3). In contrast, $\mathrm{SiO}$ is only found in the outflows, but because IRAS $05358+3543 \mathrm{~mm}$ exhibits at least three outflows, the single-dish map looks very similar to the $\mathrm{H}^{13} \mathrm{CO}^{+}$image.

The situation is different with regard to the thermal $\mathrm{CH}_{3} \mathrm{OH}$ emission at $241 \mathrm{GHz}$. While the line center emission corresponds to the dust emission, the wing emission seems to be associated with outflow $(\mathcal{C})$. From these data it appears that $\mathrm{CH}_{3} \mathrm{OH}$ is a tracer of outflows as well as of core emission.

\section{Discussion}

\subsection{The $1 p c$, highly collimated outflow $(\mathcal{A})$}

The eastern large scale outflow $(\mathcal{A})$ in IRAS $05358+3543 \mathrm{~mm}$ is the first example of a highly collimated, jet-like, bipolar and massive outflow with an extension of $\approx 1 \mathrm{pc}$. The collimation factor 10 is as high as the highest found in low-mass flows. High degrees of collimation are difficult to explain by stellar winds or deflection of infalling matter. Outflow models involving highly collimated jets entraining the surrounding material are much more likely (Cabrit et al. 1997). Churchwell (2000) argues that the predictions of shock-entrainment models for the amount of material that can be entrained are far too low to account for several tens of solar masses found in massive outflows, as it is the case for IRAS $05358+3543 \mathrm{~mm}$ (Table 2). But he also stresses the caveats in these estimates, because they are highly sensible to different assumptions, especially the entrainment efficiency in the dense interstellar medium as found in massive star formation sites. It is well possible that the entrainment efficiency rises significantly in the very dense interstellar medium, and higher efficiencies could easily account for the observed high outflow masses found in massive star-forming regions. Therefore, outflow $(\mathcal{A})$ links massive outflow phenomena to scenarios already known from low-mass star formation. It strongly suggests that entrainment by bow shocks propagating in a collimated jet also has an important role in the formation of massive outflows. 


\subsection{The high-velocity outflow (B) and the quadrupolar structure}

Emanating at a $\mathrm{PA}$ of $\approx 40^{\circ}$, most likely from the same $\mathrm{mm}$ core mm1 (Fig. 7), the less extended high-velocity outflow $(\mathcal{B})$ is clearly distinct from the large scale outflow $(\mathcal{A})$. Different quadrupolar outflow mechanisms are discussed in the literature (for a brief compilation and discussion see Gueth et al. 2001), and the most appealing explanation in the case of IRAS $05358+3543 \mathrm{~mm}$ seems to be that the two outflows are produced independently by adjacent protostars inside the same $\mathrm{mm}$ condensation mm1. Being parts of one and the same outflow is unlikely, mainly because the large scale outflow does not show any elongation at a PA of $40^{\circ}$, which would be expected if the quadrupolar structure was due to deflected or precessing material. Assuming half of the $\operatorname{HPBW}\left(\approx 1.75^{\prime \prime} \approx 3100 \mathrm{AU}\right)$ at a distance of $1.8 \mathrm{kpc}$ as the maximal separation of the two powering sources, this is well within the range of typical binary separation.

\subsection{The SiO outflow (C)}

In the bent interpretation, the $\mathrm{SiO}$ outflow $\left(\mathcal{C}_{\text {bent }}\right)$ closely follows the north-south elongation of the dust continuum emission. It is slightly less collimated than the large $\mathrm{CO}$ outflow $(\mathcal{A})$, and blue and red lobes are separated in $\mathrm{SiO}$ as well as in CO. The northern and southern lobes show three peaks each that are symmetric with respect to the assumed origin, suggesting three different ejection events. In contrast to this, the cavity interpretation of outflow $\left(\mathcal{C}_{\text {cavity }}\right)$ does not imply that the outflow has to follow the core structure, but rather that $\mathrm{SiO}$ is only observed in that part of the flow where the densities are high enough to excite $\mathrm{SiO}$ sufficiently. As already mentioned, outflow $\left(\mathcal{C}_{\text {cavity }}\right)$ then is likely to be located at the front side of the core outlined in Fig. 1. The bullet-like structures and their interpretation as three ejection events are independent of the exact scenarios.

It is a rather intriguing fact that $\mathrm{SiO}$, which forms in shocks from dust disruption (Schilke et al. 1997), is only found in regions with strong dust emission (Fig. 4). Outflow $(\mathcal{C})$ follows to a large degree the north-south dust filament, while the eastern large scale $\mathrm{CO}$ outflow leaves the dense core very soon. Thus, throughout most parts of outflow $(\mathcal{A})$, column densities are low and the gas densities there are not high enough to excite the $\mathrm{SiO}$ sufficiently. Another reason may be that that C-shocks (shock velocities $v_{\mathrm{s}} \leq 50 \mathrm{~km} \mathrm{~s}^{-1}$ ) are needed to produce SiO (Schilke et al. 1997). In the less massive outflow $(\mathcal{C})$ C-shocks are likely to be more common than in the more massive outflow $(\mathcal{A})$, where higher jet velocities are expected (>500 $\mathrm{km} \mathrm{s}^{-1}$, e.g., Eislöffel et al. 2000) and shocks are more likely of $J$-type $\left(v_{\mathrm{s}} \geq 50 \mathrm{~km} \mathrm{~s}^{-1}\right)$, dissociating the molecular material. These different scenarios have to be checked for a larger sample.

\subsection{The large scale bending}

Assuming the bent interpretation of outflow $\left(\mathcal{C}_{\text {bent }}\right)$, both large-scale outflows and the mm dust core show the same bent morphology. For the cavity interpretation of outflow $\left(\mathcal{C}_{\text {cavity }}\right)$, still outflow $(\mathcal{A})$, the southern lobe of outflow $\left(\mathcal{C}_{\text {cavity }}\right)$, and the mm dust emission exhibit the same bent structure. Possible bending mechanisms for protostellar jets are discussed, e.g., in Fendt \& Zinnecker (1998).

Internal bending scenarios on small scales seem to be ruled out in the case of $I R A S 05358+3543 \mathrm{~mm}$, because it is not likely that in both large scale outflows exactly the same small scale physics takes place (e.g., acceleration of the jet source by a binary component, or precession of an accretion disk in a binary system), and additionally the dust core follows a similar morphology.

Considering the whole source structure, external effects seem to be more plausible to explain the structure of this massive star-forming site. On larger scales (4 to 10 pc) other HiI regions are found: in the north-east S233 (at a distance of $\approx 4 \mathrm{pc}$ ), in the west S235 (at a distance of $\approx 10 \mathrm{pc}$ ), in the north-west S231 (distance $\approx 4 \mathrm{pc}$ ) and a bit further away $\mathrm{S} 232$ (distance $\approx 15 \mathrm{pc}$, for large scale images see, e.g., Porras et al. 2000). As a whole, the region contains different massive star formation sites at the edge of IRAS $05358+3543 \mathrm{~mm}$, and the most likely explanation of the overall bending of this youngest site is due to energy input (UV radiation as well as stellar winds) from the more evolved star forming regions in the vicinity. Thus, we are possibly observing an example of sequential star formation and interactions of regions of different ages.

\subsection{Outflows from young stellar objects of all masses}

A key question triggering this observational study was whether massive outflows are generated by different physical mechanism than their low-mass counterparts. We therefore compare IRAS $05358+3543 \mathrm{~mm}$ with other outflow sources of different core masses.

A low-mass object: a prototype of a low-mass outflow is HH211 emanating from a dust condensation of $\sim 0.2 M_{\odot}$ (Gueth \& Guilloteau 1999). It includes a highly collimated, high-velocity molecular jet surrounded by a less collimated cavity-like outflow at lower velocity. A shockentrainment model nicely explains the outflow structure.

An intermediate-mass object: Gueth et al. (2001) recently observed the intermediate-mass young stellar object powering HH288 (core mass between 6 and $30 M_{\odot}$ ). This outflow is quadrupolar and most likely due to two powering sources not separable by the resolution of the observation. But in spite of being quadrupolar, the whole system can be explained by two outflows with shock-entrained material similar to $\mathrm{HH} 211$.

A high-mass object: our observations of IRAS $05358+3543 \mathrm{~mm}$ show an example of a massive star-forming cluster. High-resolution Plateau de Bure observations resolve the single-dish features into a number of different outflows. So far, just a few massive 
outflows have been mapped with high spatial resolution (see Introduction), and we stress that the large scale eastern outflow is the first massive outflow observed with such a high degree of collimation (collimation factor $~ 10$ ). We estimate the accretion rate to be $10^{-4} M_{\odot} \mathrm{yr}^{-1}$, and Beuther et al. (2002b) recently showed that mass entrainment rates of massive star-forming regions are usually of that order. This is high enough to overcome the radiation pressure of the central object and build up more massive stars (Wolfire \& Cassinelli 1987; Jijina \& Adams 1996; Norberg \& Maeder 2000; Tan \& McKee 2002; Yorke 2002). Additionally, jet models require disks, which are believed to be observed in a few massive objects (e.g., IRAS 20126+4104, Cesaroni et al. 1997, Zhang et al. 1998; IRAS 23385+6053, Molinari et al. 1998). Thus, to explain the outflow features observed in IRAS $05358+3543 \mathrm{~mm}$, shock-entrainment models are sufficient (e.g., Gueth \& Guilloteau 1999; Richer et al. 2000; Cabrit et al. 1997), and no other formation mechanism is needed. It cannot be ruled out that in other sources different physical mechanisms are taking place, but these observations indicate that other massive star formation sites of rather chaotic appearance might be disentangled into simpler structures if they are observed at higher angular resolution.

\section{Summary}

IRAS $05358+3543 \mathrm{~mm}$ is the first example of a massive $\left(>10 M_{\odot}\right)$ bipolar outflow with a high degree of collimation on scales of 1 pc (collimation factor $\sim 10$ ). High-angular-resolution observations with the Plateau de Bure Interferometer resolve the single-dish observations into at least three different outflows. Two of them form a quadrupolar system, most likely emanating from adjacent protostars within the same mm core. Our data resolve three massive mm cores (between $100 M_{\odot}$ and $75 M_{\odot}$ ) at the center of the quadrupolar outflow.

The data suggest that the physical processes associated with this massive outflow are similar to those driving their low-mass counterparts. It is likely that many massive star formation sites can be shown by interferometric highresolution observations to be composed of basic features known from low-mass star formation. The accretion rate is high $\left(\sim 10^{-4} M_{\odot} \mathrm{yr}^{-1}\right)$ and consistent with disk-accretion scenarios explaining the formation of massive stars (e.g., Wolfire \& Cassinelli 1987; Jijina \& Adams 1996; Norberg \& Maeder 2000; Tan \& McKee 2002; Yorke 2002).

The overall distribution of $\mathrm{H}^{13} \mathrm{CO}^{+}, \mathrm{SiO}$ and thermal $\mathrm{CH}_{3} \mathrm{OH}$ looks very similar, but the higher resolution $\mathrm{PdBI}$ observations show varying morphologies traced by the different lines. While $\mathrm{SiO}$ is observed mainly in the outflows, $\mathrm{H}^{13} \mathrm{CO}^{+}$traces core-like structures, which do not coincide exactly with the dust cores because of varying relative $\mathrm{H}^{13} \mathrm{CO}^{+}$abundances. Finally, $\mathrm{CH}_{3} \mathrm{OH}$ can be decomposed into a core tracing component at the line center and wing emission tracing the outflows.
Ratio maps between CO 6-5, 2-1 and 1-0 reveal local temperature gradients. At the tip of the high-velocity outflow $(\mathcal{B})$, we find a temperature increase $(\geq 80 \mathrm{~K})$ caused by shock-interaction of the outflow with the ambient medium. Additionally, the southern lobe of the third outflow $(\mathcal{C})$ is much warmer (again $\geq 80 \mathrm{~K}$ ) than the rest of the outflow, possibly due to UV heating of a close by and more evolved cluster. Contrasting with these temperature increases, the possible center $\mathrm{H}^{13} \mathrm{CO}^{+}[3]$ of outflow $(\mathcal{C})$ is cold, below $15 \mathrm{~K}$.

Acknowledgements. We would like to thank the unknown referee for helpful comments on the initial draft of this paper. H. Beuther is supported by the Deutsche Forschungsgemeinschaft, DFG project number SPP 471.

\section{References}

Bonnell, I., Bate, M., Clarke, C., \& Pringle, J. 1997, MNRAS, 285, 201

Bonnell, I., Bate, M., \& Zinnecker, H. 1998, MNRAS, 298, 93

Beuther, H., Kramer, C., Deiss, B., \& Stutzki, J. 2000, A\&A, 362, 1109

Beuther, H., Sridharan, T. K., Schilke, P., Menten, K. M., \& Wyrowski, F. 2002a, ApJ, 566, 945

Beuther, H., Schilke, P., Sridharan, T. K., et al. 2002b, A\&A, 383, 892

Beuther, H., Walsh, A., Schilke, P., et al. 2002c, A\&A, submitted

Cabrit, S., \& Bertout, C. 1990, ApJ, 348, 530

Cabrit, S., Raga, A., \& Gueth, F. 1997, in IAU Symp., 182

Canto, J., \& Raga, A. C. 1991, ApJ, 372, 646

Cesaroni, R., Felli, M., Testi, L., Walmley, C. M., \& Olmi, L. 1997, A\&A, 325, 725

Cesaroni, R., Felli, M., Jenness, T., et al. 1999, A\&A, 345, 949

Chan, S., Henning, T., \& Schreyer, K. 1996, A\&AS, 115, 285

Choi, M., Evans, N. J. II, \& Jaffe, D. T. 1993, ApJ, 417, 624

Churchwell, E. 2000, in The Origins of Stars and Planetary Systems, ed. C. J., Lada, \& N. D. Kylafis (Kluwer Academic Press)

Eislöffel, J., Mundt, R., Ray, T. P., \& Rodriguez, L. F. 2000, in ProtoStars \& Planets IV, ed. V. Mannings

Fendt, C., \& Zinnecker, H. 1998, A\&A, 334, 750

Frerking, M., Langer, L., \& Wilson, R. 1982, ApJ, 262, 590

Gueth, F., Guilloteau, S., \& Bachiller, R. 1996, A\&A, 307, 891

Gueth, F., \& Guilloteau, S. 1999, A\&A, 343, 571

Gueth, F., Schilke, P., McCaughrean, M., et al. 2001, A\&A, 375,1018

Guilloteau, S., Delannoy, J., Downes, D., et al. 1992, A\&A, 323,943

Hildebrand, R. 1983, QJRAS, 24, 267

Hirano, N., \& Taniguchi, Y. 2001, ApJ, L219

Hunter, T. 1997, Ph.D. Thesis, Caltech

Jiang, Z., Yao, Y., Yang, J., et al. 2001, AJ, 122, 313

Jijina, J., \& Adams, F. 1996, ApJ, 462, 874

Kurtz, S., Cesaroni, R., Churchwell, E., \& Walmsley, C. M. 2000, in ProtoStars \& Planets IV, ed. V. Mannings

Langer, W., \& Penzias, A. 1990, ApJ, 357, 477

Lis, D., Serabyn, E., Keene, J., et al. 1998, ApJ, 509, 299

McCaughrean, M. J., Stanke, T., \& Andersen, M. 2002, A\&A, in preparation

Menten, K. 1991, ApJ, 380, L75 
Mezger, P., Wink, J., \& Zylka, R. 1990, A\&A, 228, 95

Molinari, S., Testi, L., Brand, J., Cesaroni, R., \& Palla, F. 1998, ApJ, 505, L39

Molinari, S., Brand, J., Cesaroni, R., \& Palla, F. 2000, A\&A, 355,617

Norberg, P., \& Maeder, A. 2000, A\&A, 359, 1025

Porras, A., Cruz-Gonzales, I., \& Salas, L. 2000, A\&A, 361, 660

Ramesh, B., \& Sridharan, T. K. 1997, MNRAS, 284, 1001, (RS)

Richer, J., Shepherd, D., Cabrit, S., Bachiller, R., \& Churchwell, E. 2000, in ProtoStars \& Planets IV, ed. V. Mannings

Schilke, P., Walmsley, C., Pineau des Forets, \& Flower, D. 1997, A\&A, 321, 293

Shepherd, D., \& Churchwell, E. 1996, ApJ, 472, 225

Shepherd, D., Watson, A., Sargent, A., \& Churchwell, E. 1998, ApJ, 507, 861

Shepherd, D., \& Kurtz, S. 1999, ApJ, 523, 690

Shu, F. 1977, ApJ, 214, 488

Snell, R., Dickman, R., \& Huang, Y. 1990, ApJ, 352, 139

Sridharan, T. K., Beuther, H., Schilke, P., Menten, K. M., \& Wyrowski, F. 2002, ApJ, 566, 931
Stahler, S., Palla, F., \& Ho, P. 2000, in ProtoStars \& Planets IV (The University of Arizona Press)

Tan, J., \& McKee, C. 2002, Proceedings of The earliest stages of massive star formation, to be published in ASP Conf. Ser., ed. P. Crowther

Synthesis Imaging in Radio Astronomy II, 1999, ASP Conf. Ser., 180, ed. G. B. Taylor, C. L. Carilli, \& R. A. Perley

Tofani, G., Felli, M., Taylor, G., \& Hunter, T. 1995, A\&AS, 112,299

van Dishoeck, E. F., Blake, G. A., Draine, B. T., \& Lunine, J. I. 1993, in ProtoStars \& Planets III (The University of Arizona Press)

Walmsley, M. 1995, RMxAC, 1, 137

Wolfire, M., \& Cassinelli, J. 1987, ApJ, 319, 850

Wouterloot, J., Brand, J., \& Henkel, C. 1988, A\&A, 191,323

Yao, Y., Ishii, M., Nagata, T., Nakaya, H., \& Sato, S. 2000, ApJ, 542, 392

Yorke, H. 2002, Proceedings of The earliest stages of massive star formation, to be published in ASP Conf. Ser., ed. P. Crowther

Zhang, Q., Hunter, T. R., \& Sridharan, T. K. 1998, ApJ, 505, L151 